\title{
Nano-encapsulated tarragon (Artemisia dracunculus) essential oil as a sustained release nano-larvicide
}

\author{
Mahmoud Osanloo, a,b Mohammad Mehdi Sedaghat, ${ }^{c}$ Hassan Sereshti, ${ }^{d}$ and Amir Amani ${ }^{\text {e,* }}$
}

\author{
${ }^{a}$ Department of Medical Nanotechnology, School of Advanced Technologies in Medicine, Tehran University of Medical Sciences, Tehran, Iran. \\ bepartment of Medical Nanotechnology, School of Advanced Technologies in Medicine, Fasa University of Medical Sciences, Fasa, Iran. \\ 'Department of Medical Entomology and Vector Control, School of Public Health, Tehran University of Medical Sciences, Tehran, Iran. \\ dDepartment of Chemistry, Faculty of Science, University of Tehran, Tehran, Iran. \\ eNatural Products and Medicinal Plants Research Center, North Khorasan University of Medical Sciences, Bojnurd, Iran. \\ 'Medical Biomaterials Research Center, Tehran University of Medical Sciences, Tehran, Iran. \\ ${ }^{*}$ Correspondence to Amir Amani (email: aamani@sina.tums.ac.ir). \\ (Submitted: 03 January 2019 - Revised version received: 20 January 2019 - Accepted: 11 February 2019 - Published online: 26 April 2019)
}

\begin{abstract}
Objective In recent years, essential oil-based larvicides have been introduced as alternatives to industrial ones. However, an appreciable formulation of essential oils with prolonged larvicidal activity $(L A)$ has not yet been developed.

Methods In this study, tarragon essential oil (TEO) was encapsulated in chitosan nanoparticles using ion gelation technique. Physicochemical properties and duration of LA of the prepared nanoformulation were investigated.

Results Encapsulation efficiency of the optimum nanoformulations with a particle size of $168 \pm 90 \mathrm{~nm}$ was calculated as $39.66 \%$ using UV-Vis analysis. Encapsulating TEO in chitosan-tripolyphosphate nanocapsules was shown to increase its efficiency in LA at SSF test: Perfect LA (100\% mortality) was achieved at lower concentration (i.e. $31 \mu \mathrm{g} / \mathrm{mL}$ instead of $80 \mu \mathrm{g} / \mathrm{mL}$ ). Also, perfect LA continued for 4 and 3 days, compared with 2 days and 1 day for the non-encapsulated form of TEO in the lab and SFF tests, respectively. Besides, the duration of LA of nanoformulation was significantly longer than its corresponding microformulation with the same concentration of ingredients. Furthermore, the concentration of TEO in the solution tests was also monitored and it was found that the nanoformulations provide a sustained release of TEO. Moreover, there was a logical relationship between LA and concentration of TEO in different hours.

Conclusion This prepared nanoformulation could be introduced as an interesting alternative to synthetic larvicides, due to its easy and fast method of preparation and its green constituents.

Keywords nanoencapsulation, tarragon essential oil, larvicidal activity, Anopheles stephensi, chitosan nanocapsules
\end{abstract}

\section{Introduction}

Malaria is still regarded as a global public health problem: an estimated death of 429,000 was caused by malaria just in the year 2015, with transmission occurring in 91 countries around the world. ${ }^{1}$ Controlling mosquito larvae, especially in the endemic region, is probably the easiest and the best costeffective way to control mosquito-borne diseases and eliminate disease transmission. ${ }^{2-4}$ However, occurring resistance in mosquitoes, environmental pollution, and adverse effects on non-target species have been observed due to the continuous use of synthetic larvicides. In case of resistance, a study by Peiris et al. ${ }^{5}$ was one of the first reports on the occurrence of resistance in mosquitoes (i.e. Culex quinquefasciatus) against Temephos. Since then, several reports on the occurrence of resistance in other mosquitoes population have been published e.g. in Aedes aegypti, ${ }^{6-8}$ and Anopheles stephensi. ${ }^{9-11}$

To overcome the mentioned problems, encapsulations of active agents have been proposed. Generally, by encapsulation of active components and providing a sustained release, two effects are expected: reduced side effects and prolonged activity. ${ }^{12-14}$ For example, encapsulation of Temephos in polyethylene glycol (PEG), and chitosan/alginate/gelatin against Culex spp. led to the reduction of side effects on non-target species and environment by controlling the release of the larvicide. ${ }^{15,16}$

An interesting alternative for synthetic larvicides is the use of plant-derived essential oil/extraction. Lethal concentration $50 \%\left(\mathrm{LC}_{50}\right)$ has been reported for many essential oils such as Citrus aurantium ( $\mathrm{LC}_{50}: 31.20 \mu \mathrm{g} / \mathrm{mL}$ ), Citrus paradise
$\left(\mathrm{LC}_{50}: 35.71 \mu \mathrm{g} / \mathrm{mL}\right)$ and Kelussia odoratissima $\left(\mathrm{LC}_{50}: 4.77\right.$ $\mu \mathrm{g} / \mathrm{mL}$ ) against $A$. stephensi. ${ }^{17,18}$ Besides, LC $_{50}$ of leaf extracts of Acanthospermum hispidum and Cleistanthus collinus against A. stephensi were reported as 20.96 and $67.20 \mu \mathrm{g} / \mathrm{mL}$, respectively. ${ }^{19,20}$

However, the main disadvantage of plant-derived essential oils is their very short shelf-life due to evaporation. Thus, many attempts have been made to encapsulate essential oils for the control of insects both in agriculture (pests) or urban and rural life (vermin and vectors). For instance, PEG nanocapsules containing Garlic essential oil showed better mortality (80\%) compared with the free EO (11\%) after 5 months against Tribolium castaneum. ${ }^{21}$ However, no work so far has reported of a long-lasting larvicidal activity (LA) in aqueous media when nanoformulations of essential oil have been prepared. ${ }^{22-26}$

Artemisia dracunculus, known as tarragon belongs to Asteraceae family; different properties of its essential oil/ extract were confirmed in recent years. For example, adjusting the level of blood glucose ${ }^{19,20}$ or the inhibition of blood platelet adhesion. ${ }^{27,28}$ In this study, for the first time, a sustained release nanoformulation of tarragon essential oil (TEO) has been introduced and its release profile has been monitored. Furthermore, by encapsulating the TEO in chitosan nanocapsules, we tried to overcome the volatility of TEO and increasing continuity of LA. Also, LA of nanoformulation was compared with microformulation, with the same concentration of ingredients. 


\section{Materials and Methods}

\section{Materials}

Chitosan $\left(M_{\mathrm{w}}=100 \mathrm{kD}, \mathrm{DD}=93 \%\right)$ was bought from Easter Holding Group (China), TEO was purchased from Zardband Pharmaceuticals Co (Iran), stored at $4-6^{\circ} \mathrm{C}$, away from sunlight. Tween 20 (TW), ethanol (Eth), tripolyphosphate (TPP) and acetic acid were supplied from Merck Chemicals (Germany).

\section{Evaluation of Larvicidal Properties of TEO and Selected Nanoformulations}

Larvicidal activity of TEO in the lab test was performed in our previous research $\left(\mathrm{LC}_{50}\right.$ and $\mathrm{LC}_{90} ; 11.36$ and $17.54 \mu \mathrm{g} / \mathrm{mL}$, respectively). ${ }^{29}$ In this study, LA of TEO was evaluated under simulated semi-field (SSF) condition i.e. $34 \pm 6^{\circ} \mathrm{C}$ with $12: 12$ light and dark photoperiods and $25 \pm 10 \%$ relative humidity, comparable with temperature and humidity in a shaded location, during the hot season of Tehran (Research location).

Furthermore, the duration of LA of each of the selected nanoformulations (i.e. F1, F2, and F3) was compared with similar concentrations of TEO dissolved in Eth (bulk TEO), in both lab and SSF tests in line with WHO guidelines with some modifications. ${ }^{30}$ It should be noted that lab investigations were performed under recommended conditions (i.e. $28 \pm 1^{\circ} \mathrm{C}$ with 12:12 light and dark photoperiods and $65 \pm 5 \%$ relative humidity).

For both types of larvicidal bioassays (i.e. lab and SSF), laboratory-reared $3^{\text {rd }}$ and $4^{\text {th }}$ instar larvae of $A$. stephensi were obtained from the department of medical entomology, Tehran University of Medical Sciences. Tests were repeated 12 times in three different replicates. In each replicate, two control groups were considered; having $1 \mathrm{~mL}$ of $E^{\text {th }}$ and nanoformulation without TEO [F(-oil)]. During the tests, containers were isolated from the environment with help of nets, to prevent environment mosquitoes mixing with larvae of the tests.

\section{Determining a Diagnostic Dose of TEO in SSF Test}

Bulk TEO stock solution $(20 \mu \mathrm{L} / \mathrm{mL})$ was prepared by dissolving and diluting with $E^{\text {th }}$ to prepare working standard solutions. One $\mathrm{mL}$ of working standard solutions was added to containers with $199 \mathrm{~mL}$ of no chlorine water to obtain bulk TEO concentrations of $20,40,80$ and $100 \mu \mathrm{g} / \mathrm{mL}$ in each container. Containers solution was mixed. Then, batches of 25 larvae were added to each container. After $24 \mathrm{~h}$ of exposure, dead larvae were counted. The lowest concentration of bulk TEO that had perfect LA (100\%) was selected as the diagnostic dose for SSF test.

\section{Evaluating the Continuity of LA of Selected Nanoformulation in Lab and SSF Tests}

In summary, batches of 25 larvae were added to containers having $199 \mathrm{~mL}$ with no chlorine water. By adding $1 \mathrm{~mL}$ from bulk TEO or nanoformulations (i.e. F1, F2, and F3), TEO concentration in each container was eventually fixed at 18,50 or $80 \mu \mathrm{g} / \mathrm{mL}$. After $24 \mathrm{~h}$ of exposure and counting the dead larvae, all the larvae (both dead and live) were removed using rubber pipette and other batches of 25 live larvae were added to the containers. The tests were stopped when LA of containers equalled that of containers having controls. The tests were discarded when mortality in control groups (containing $E^{\text {th }}$ ) increased to $5 \%$, to ensure the accuracy of the test.

\section{Preparation of Chitosan Nanocapsules Containing TEO}

From the larvicidal bioassays on bulk TEO, three different concentrations of TEO were selected for encapsulating in chitosan nanocapsules. The lowest amount of TEO (i.e. $0.36 \%$ ) was comparable with $\mathrm{LC}_{90}$ of bulk TEO in the lab test and highest amount (i.e. 1.60\%) was equal to the concentration of bulk TEO having perfect LA in SSF test. A third concentration was also studied as a value between the two values mentioned above (i.e. $1.00 \%$ ).

To prepare chitosan nanocapsules containing TEO, ion gelation technique with some modifications was used. ${ }^{31}$ Briefly, stock solutions of chitosan were prepared by dissolving chitosan $(3 \% \mathrm{w} / \mathrm{w})$ in an aqueous solution of acetic acid $(1 \% \mathrm{w} / \mathrm{w})$. Different working aqueous solutions of chitosan (i.e. $0.05,0.1,0.2,0.4$, and $0.8 \%$ ) were prepared by dilution of stock solution with acetic acid $(1 \% \mathrm{w} / \mathrm{w})$. After mixing TEO, TW and $E^{\text {th }}$ (600 rpm, room temperature, and $15 \mathrm{~min}$ ), working chitosan solutions were added dropwise, for preparing a homogenized mixture. For the formation of chitosan nanocapsules containing TEO, aqueous solutions of TPP (i.e. $0.02,0.04,0.07$, and $0.1 \% \mathrm{w} / \mathrm{w}$ ) were added to each mixture at once. The mixtures were then stirred for $30 \mathrm{~min}(1800 \mathrm{rpm})$ at room temperature.

It should be noted that, at each concentration of TEO, a fixed amount of TW and $E^{\text {th }}$ was used to form a homogeneous mixture under the mentioned condition $(600 \mathrm{rpm}$, room temperature, and $15 \mathrm{~min})$. By using TEO 0.36\%, TW $2.5 \%$ and $E^{\text {th }}$ $7.14 \%$ was used. However, at TEO $1.00 \%$ : TW and $E^{\text {th }} 3.00$ and $6.00 \%$, were used respectively. Moreover, TW $2.80 \%$ and $E^{\text {th }}$ $5.80 \%$ were added using TEO $1.6 \%$.

\section{Characterization of the Nanoformulations}

Particle size (PS) and particle size distribution (PSD) of the formulations were determined using dynamic light scattering (DLS, scatteroscope-I, K-ONE, Korea), and confirmed by transition electron microscopy (TEM, LEO 906E Zeiss, Germany). D50, as reported by the DLS instrument, was taken as PS. PSD was calculated using Eq. (1). Measuring the size of the nanocapsules samples was repeated at three different times and the mean \pm standard deviation was reported as the final size.

$$
\operatorname{PSD}=\sqrt{\frac{d 75}{d 25}}
$$

In each of the three concentrations of TEO, one formulation with the smallest size (named F1, F2, and F3) was selected for further investigation such as characterization and larvicidal bioassays. For determining the encapsulation efficiency of the selected nanoformulations, the samples were centrifuged under defined conditions (i.e. $4^{\circ} \mathrm{C}, 17700 \mathrm{~g}$ and 60 min). By determining the amount of TEO in the supernatant using UV-Vis spectroscopy (CE 7250 Double Beam Spectrophotometer, Cecil, UK) and deducing it from initial amount, using a specific regression equation, encapsulation efficiency was calculated [see Eq. (2)]. Nanoformulations without TEO $[\mathrm{F}(-\mathrm{oil})]$ were also prepared and used as blank samples.

$$
\begin{aligned}
& \begin{array}{l}
\text { Encapsulation } \\
\text { efficiency }(\%)
\end{array}=\frac{\begin{array}{l}
\text { Initial amount of TEO }- \\
\text { The amount of TEO in supernatant }
\end{array}}{\text { Initial amount of TEO }} \times 100
\end{aligned}
$$


To determine the weight of residue after centrifugation of formulation, after discarding the supernatant, upside down falcons containing the residue and were kept at $70^{\circ} \mathrm{C}$ for 30 min to evaporate its moisture. Using Eq. (3), the loading capacity of selected nanoformulations was calculated. Determining the encapsulation efficiency and loading capacity for three selected nanoformulations were repeated at three different times and the mean \pm standard deviation was reported as the final data.

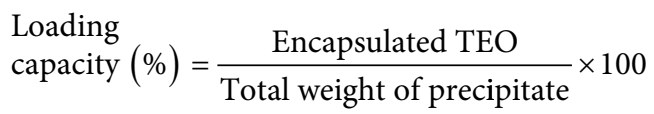

\section{Monitoring Concentration of TEO during Larviciding Test}

To investigate the reason for longer durability of LA of the optimum nanoformulation (F3) in comparison with its corresponding bulk TEO, the concentration of TEO was monitored during lab and SSF tests, using UV-Vis spectroscopy. It should be noted that F3 has been selected as optimum nanoformulation due to longer durability of LA (see Section 3.4).

\section{Evaluating the Effect of Encapsulation and Size of the Capsules in the Duration of LA}

Larvicidal activity of F3 compared with its non-encapsulated form, i.e. no TPP was added [named F3(-TPP)] to assess the effect of encapsulation of TEO in the nanoformulations. A second study was also performed to evaluate the effect of capsules size on the duration of LA. For this purpose, a formulation with similar components to that of F3, but with larger size [named F3(micro)] was also prepared using a different processing condition (i.e. dropwise addition of TPP instead of one-shot addition). Then, its LA was compared with F3.

\section{Statistical Analysis}

To compare the continuity of LA of the selected nanoformulations with each other or in comparison with related bulk TEO or results of LA in lab and SSF tests, independent-samples $t$-test with a $95 \%$ confidence interval $(95 \% \mathrm{CI})$ was used. To analyze and compare the durability of LA of the optimum nanoformulation (F3) with its related microformulation F3(micro) and non-encapsulated form F3(-TPP), one-way ANOVA (95\% CI) was used. Statistical analyses were done using SPSS (v22) software.

\section{Results and Discussion}

\section{Diagnostic Dose of TEO at SSF Larvicidal Bioassay}

The lowest concentration of bulk TEO showing perfect LA in SSF test was $80 \mu \mathrm{g} / \mathrm{mL}$ (Fig. 1). This value for the lab test was $20 \mu \mathrm{g} / \mathrm{mL}$ in our previous report. ${ }^{29}$ A reason for this difference could be extra evaporation of TEO in the SSF test due to a higher temperature, the presence of air circulation, and lower humidity compared with the lab test.

Furthermore, the ingredients of the used batch of TEO in this study were reported in our previous research. Totally, 48 components of TEO were determined by GC-MS analysis. Five major components of TEO include estragole (67.623\%), cis-ocimene (8.691\%), beta-Ocimene Y (7.577\%), limonene (4.338\%), and 3-methoxy cinnamaldehyde (1.491\%). ${ }^{29}$

\section{Evaluating the Effect of Chi and TPP Concentration on the Size of the Chitosan Nanocapsules Containing TEO}

The effect of TPP and chitosan concentrations on the final size of the nanoformulation containing fixed amounts of TEO, TW, and $E^{\text {th }}$ (i.e. $0.36,2.50$, and $7.14 \%$, respectively) are demonstrated in Fig. 2A. Samples containing high concentrations of TPP (i.e. $0.07 \%$ and $0.10 \%$ ) and low concentrations of chitosan (i.e. $0.10 \%$ and $0.05 \%$ ) started to precipitate. Thus, they were excluded from being reported in Fig. 2A. The smallest formulation with the size of $384 \pm 60 \mathrm{~nm}$ (named F1) had $0.10 \%$ and $0.04 \%$ of chitosan and TPP, respectively. From Fig. 3A, PSD of this nanoformulation was calculated as 2.2, showing a satisfactory distribution.

In Fig. 2B, the effects of concentrations of TPP/chitosan on the final size of the formulations are investigated when the amounts of TEO, TW, and $E^{\text {th }}$ are fixed at 1.00, 3.00 and $6.00 \%$, respectively. As the details show, to prepare smaller particles, a balance between concentrations of the ingredients is necessary. Nanoformulation with the smallest particle size (named F2: $116 \pm 40 \mathrm{~nm}$ ) was obtained using $0.2 \%$ and $0.04 \%$ of chitosan and TPP, respectively. From Fig. 3B, PSD of this formulation was calculated as 1.3 , an improved distribution compared with that of F1.

The effect of concentrations of TPP/chitosan on the size of nanoformulation (containing a fixed amount of TEO, TW and $E^{\text {th }}$ at $1.60,2.80$, and $5.60 \%$, respectively) are illustrated in Fig. 2C. The smallest nanoformulation (named F3) with the size of $168 \pm 90 \mathrm{~nm}$ was obtained at $0.8 \%$ and $0.04 \%$ of chitosan and TPP, respectively. From Fig. 3C: PSD of this formulation was 1.34 with good monodispersity. A TEM image of F3 depicted in Fig. 3D, shows spherical particles with a size of $155 \pm 12$.

Our previous studies indicated that decreasing the size of nanoparticles led to an increase in its efficacy. For instance, mortality of larvae, when using nanoemulsion with the size of $11 \mathrm{~nm}(92.71 \%)$, was significantly better than the corresponding emulsion with the size of $9310 \mathrm{~nm}(81.67 \%)$ at $18 \mu \mathrm{g} / \mathrm{mL}$ concentration of TEO..$^{29}$ In another study, the nanoemulsion of essential oil of Anethum graveolens with a particle size of $\sim 10 \mathrm{~nm}$, showed LA of $81 \%$, compared with the bulk form with LA of $73 \%$.

In another report, Neem nanoemulsion with three difference sizes (31, 93, and $251 \mathrm{~nm}$ ) showed maximum LA when

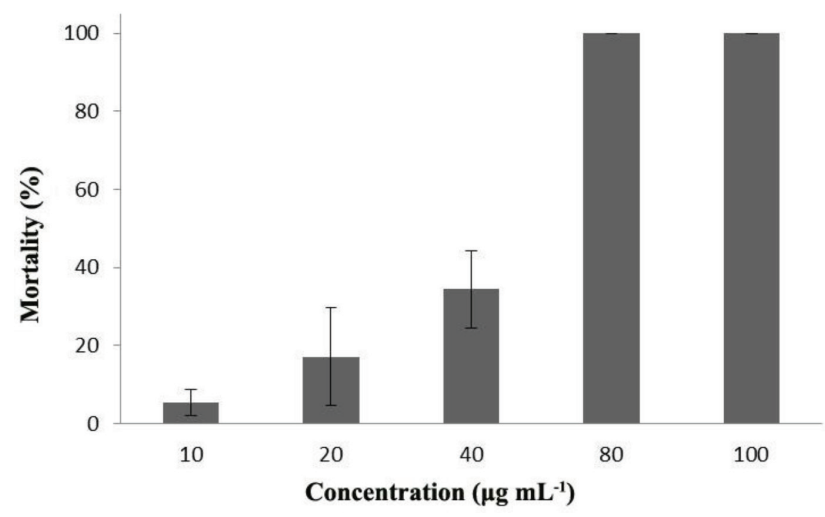

Fig. 1 Larvicidal activity of essential oil at simulated semi-field (SSF) test. 

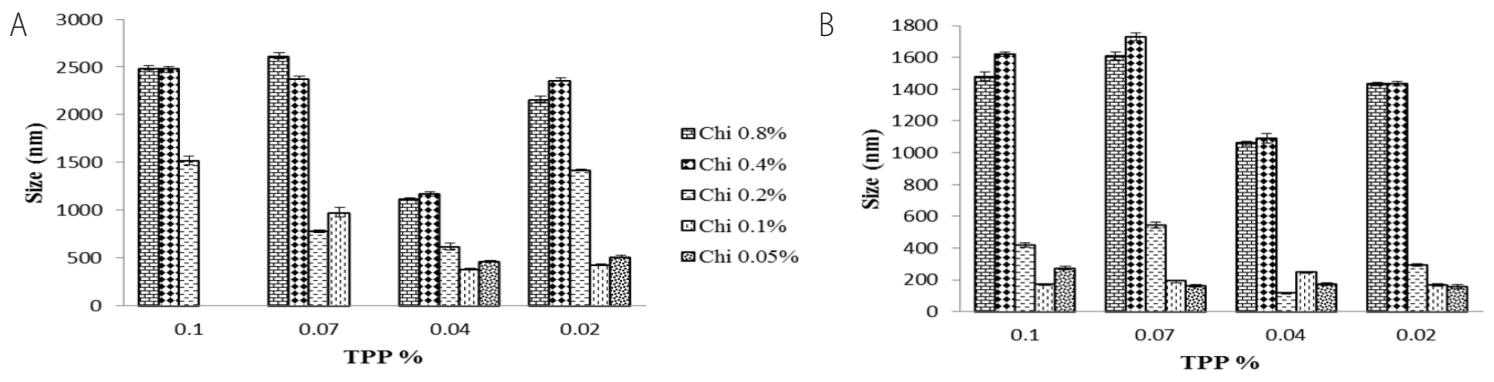

由Chi $0.8 \%$ aChi $0.4 \%$ EChi $0.2 \%$ DChi $0.1 \%$ m Chi $0.05 \%$

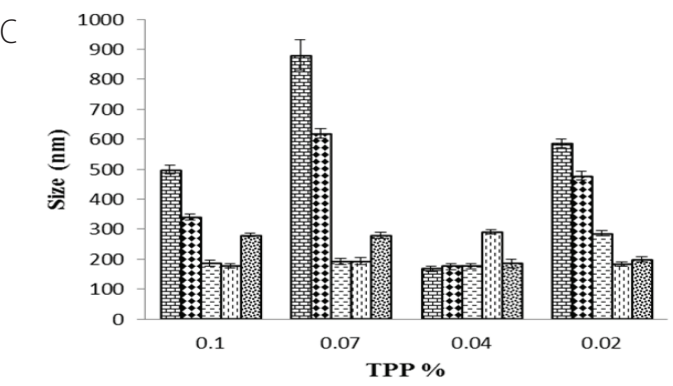

田 Chi $0.8 \%$

Chi $0.4 \%$

재i $0.2 \%$

巴. Chi $0.1 \%$

mChi $0.05 \%$

Fig. 2 Effect of TPP and chitosan concentrations on the size of the formulations containing three fixed amounts of essential oil: (A) $0.36 \%$, (B) $1.00 \%$, and (C) 1.60\%. TPP: tripolyphosphate.
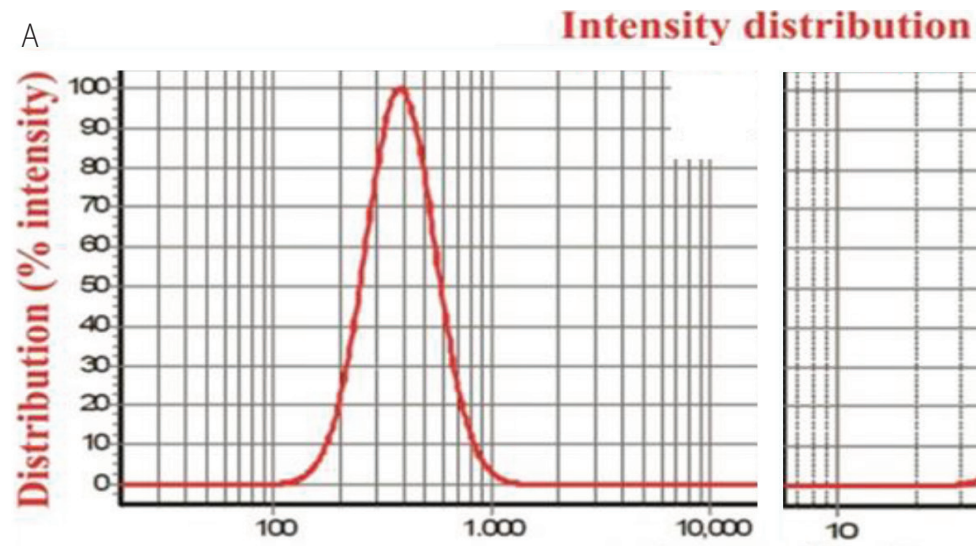

B
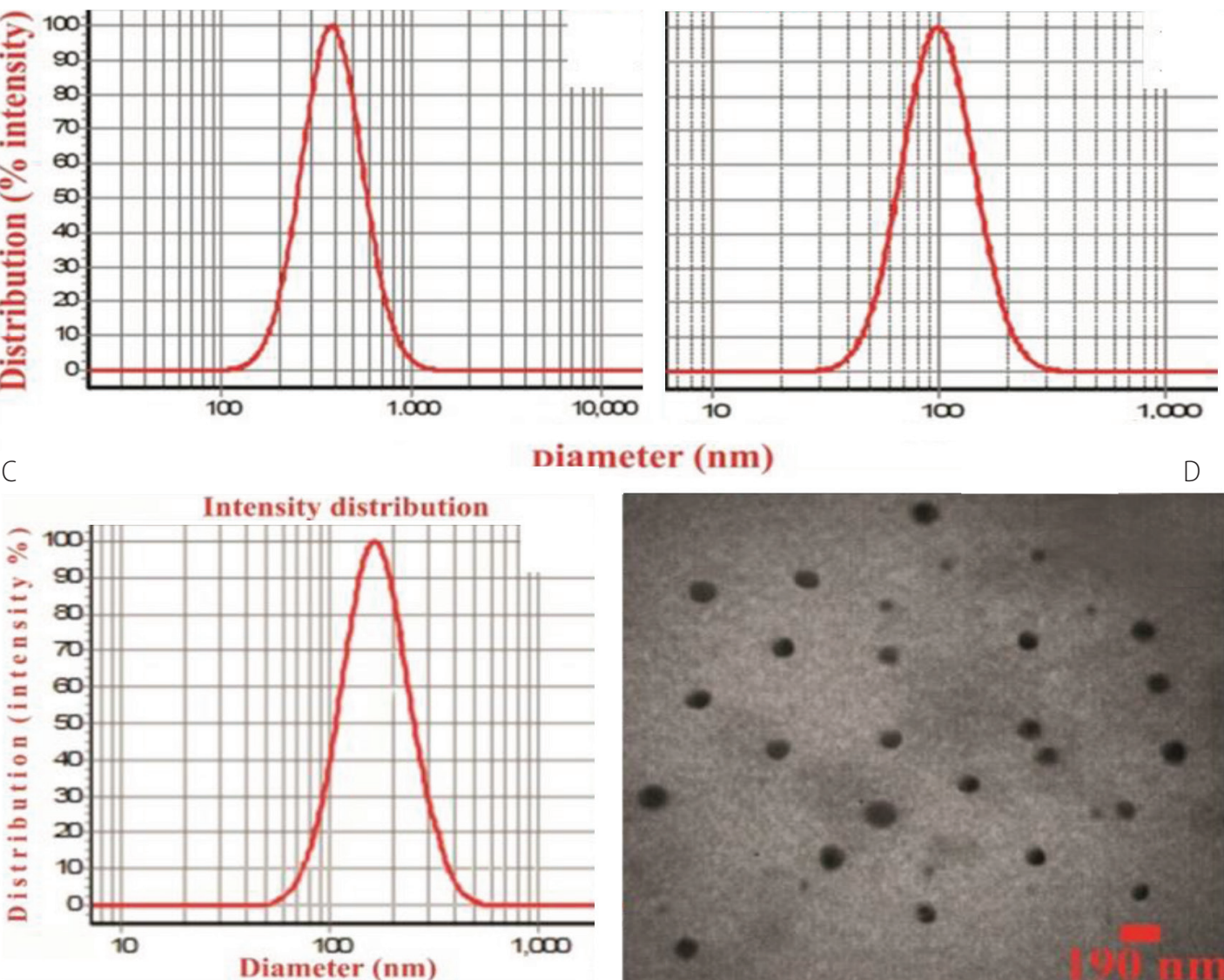

iameter $(\mathrm{nm})$

Fig. 3 DLS analysis of the selected nanoformulations: (A) F1 (384 $\pm 60 \mathrm{~nm})$, (B) F2 (116 $\pm 40 \mathrm{~nm}$ ) and (C) F3 (168 \pm 90$)$, respectively. (D) TEM image of F3 nanoformulation. DLS: dynamic light scattering, TEM: transition electron microscopy.

particle size was minimum (i.e. $31 \mathrm{~nm}$ ). ${ }^{23}$ Thus, in this study, the formulations with the smallest particle size (i.e. F1, F2, and F3) in each concentration of TEO were selected for further evaluations including characterization and larvicidal bioassays.

\section{Determining the Encapsulation Efficiency and Loading Capacity of the Selected Nanoformulations}

The results of encapsulation efficiency of the selected nanoformulations are illustrated in Table 1. Maximum encapsulation 


$\begin{aligned} & \text { Table 1. Encapsulation efficiency and loading capacity of the } \\
& \text { selected nanoformulations }\end{aligned}$
\begin{tabular}{lll}
\hline Formulations & Encapsulation efficiency (\%) & Loading capacity (\%) \\
\hline F1 & $31.21 \pm 0.90$ & $14.88 \pm 1.50$ \\
F2 & $25.10 \pm 1.00$ & $16.30 \pm 1.70$ \\
F3 & $39.66 \pm 0.90$ & $22.24 \pm 2.00$ \\
\hline
\end{tabular}

efficiency (i.e. 39\%) was for F3, probably, due to its larger chitosan content to carry TEO. Calculated regression equations and their calibration curves are demonstrated in Fig. 4A-C. Besides, one UV-Vis spectrum is also presented as an example in Fig. 4D.

The range of encapsulation efficiency in this study was close to other similar studies i.e. encapsulation of essential oils in chitosan; such as Zataria multiflora $(45.24 \%),{ }^{32}$ Carum copticum (36.2\%), ${ }^{33}$ oregano (24.72\%). ${ }^{31}$ Furthermore, loading capacity in this research was also comparable with similar studies [i.e. loading capacity values of $19.5 \%$ for C. copticum ${ }^{3.3}$ and $9.05 \%$ for Z. multiflora]. ${ }^{32}$

\section{Comparison of the Duration of LA of the Selected Nanoformulations and Bulk TEO in Lab and SSF Tests}

Figure 5A compares LA of F1 with bulk TEO having a similar concentration (i.e. $0.36 \%$ ) in the lab test. Formulation without TEO [i.e. F1(-oil)], showed no LA. Also, LA of F1 was significantly lower (independent-samples $t$-test, $P<0.05$ ) than that of bulk TEO during the first day. This could be probably due to the encapsulation of TEO which has made some of EO out of access to the larvae. However, during the second day, some LA was still observed in F1, while bulk TEO showed no LA during the same period. It is arguable that this is due to complete evaporation of bulk TEO after the first day, while the sustained release of the oil made some of it available on the second day. LA of F1 did not continue on the third day. SSF test for F1 was not performed since the formulation failed to show a perfect LA in the lab test.
The results of the comparison of LA of F2 with a similar concentration of bulk TEO (i.e. 1.00\%) in lab and SSF tests are illustrated in Fig. 5B and 5C, respectively. In the lab test, F2 and bulk TEO showed a perfect LA for 2 days and 1 day, respectively. But, observed LA for F2 was significantly higher than bulk TEO at 48,72 , and $96 \mathrm{~h}$ (independent-samples $t$-test, $P<0.05)$. After 5 days, LA of F2 did not have any significant difference (independent-samples $t$-test, $P>0.05$ ) in comparison with LA the blank sample [i.e. F2(-oil) 15\%]. Thus, the test was stopped (Data not presented). Also, Fig. 5C shows the SSF results for F2 and its corresponding bulk TEO. From the details, perfect LA was not observed in either sample. It is worth noting that LA of bulk TEO did not continue till the second day, while LA of F2 was $32 \%$ on the second day which was significantly higher than bulk TEO (independent-samples $t$-test, $P<0.05)$. This is another indication of the role of chitosan in providing a sustained release and preventing the loss of activity of TEO. No LA was observed on the third day, due to complete evaporation of TEO in the test containers.

The comparison between LA of bulk TEO (1.60\%) and corresponding encapsulated nanoformulation (i.e. F3) in both lab and SSF was made and the results are given in Fig. 5D and 5E. According to Fig. 5D, perfect LA was observed at 24 and $48 \mathrm{~h}$ in F3 and bulk TEO, but LA of F3 was significantly higher than at 72,96 , and $120 \mathrm{~h}$ (independent-samples $t$-test, $P<0.05)$ in a lab test. Furthermore, full LA of F3 in SSF test (see Fig. 5E) continued for $72 \mathrm{~h}$, while, bulk TEO showed a full activity for only one day (significantly lower than, independent-samples $t$-test, $P<0.05$ ). According to the mentioned results, the durability of LA of F3 in both lab and SSF tests was significantly higher than other nanoformulations (F1 and F2) (independent-samples $t$-test, $P<0.05)$. Thus, it was selected for further investigations as optimum nanoformulation.

Reviewing literature, slow release formulations containing essential oil of Lippia sidoides have been found. By encapsulating that essential oil in chitosan/cashew gum beads with a size of around $1.5 \mathrm{~mm}$, larvae of $A$. aegypti controlled up to 3 days. ${ }^{34}$ It was not clear what was the meaning of control, but this value was lower than that of this research i.e. 5 days.
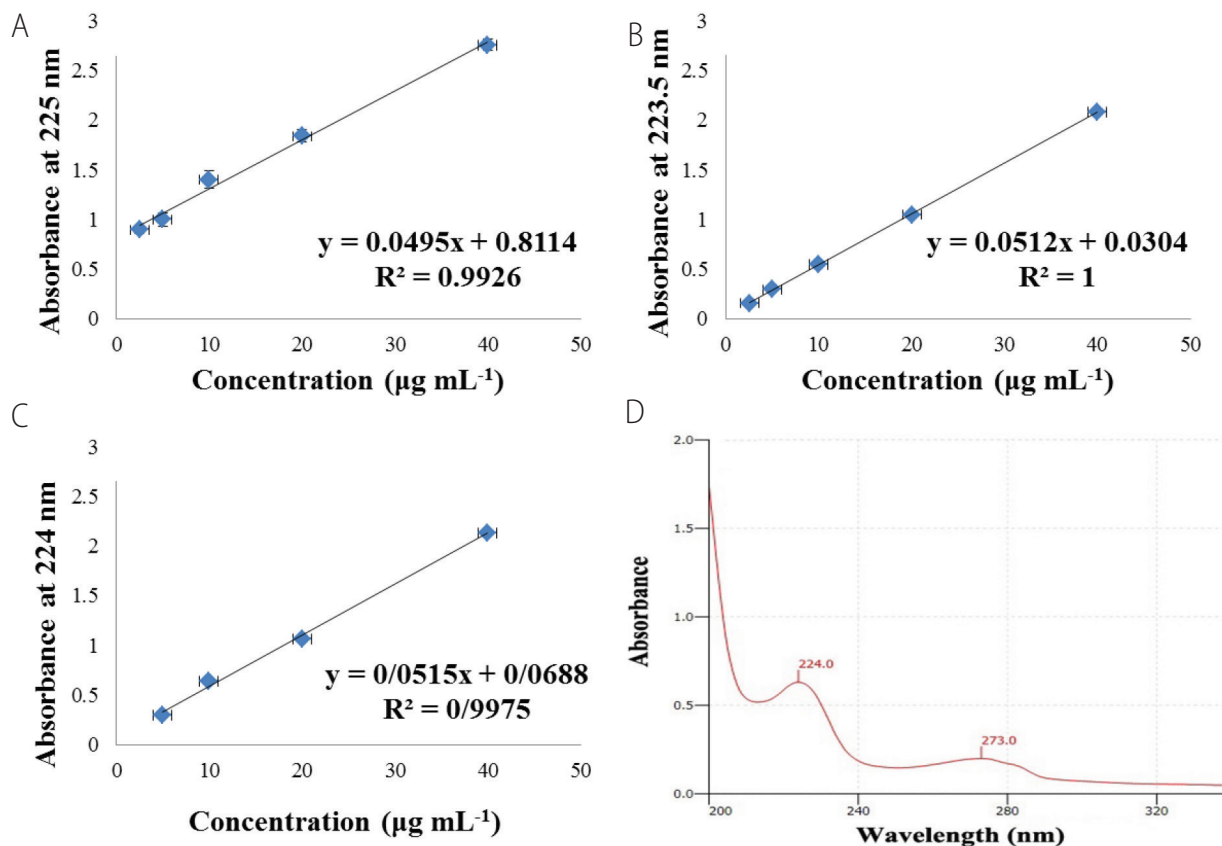

Fig. 4 Specific calibration curves and regression equations for selected nanoformulations: (A) F1, (B) F2, and (C) F3. (D) UVVis a sample spectrum. 

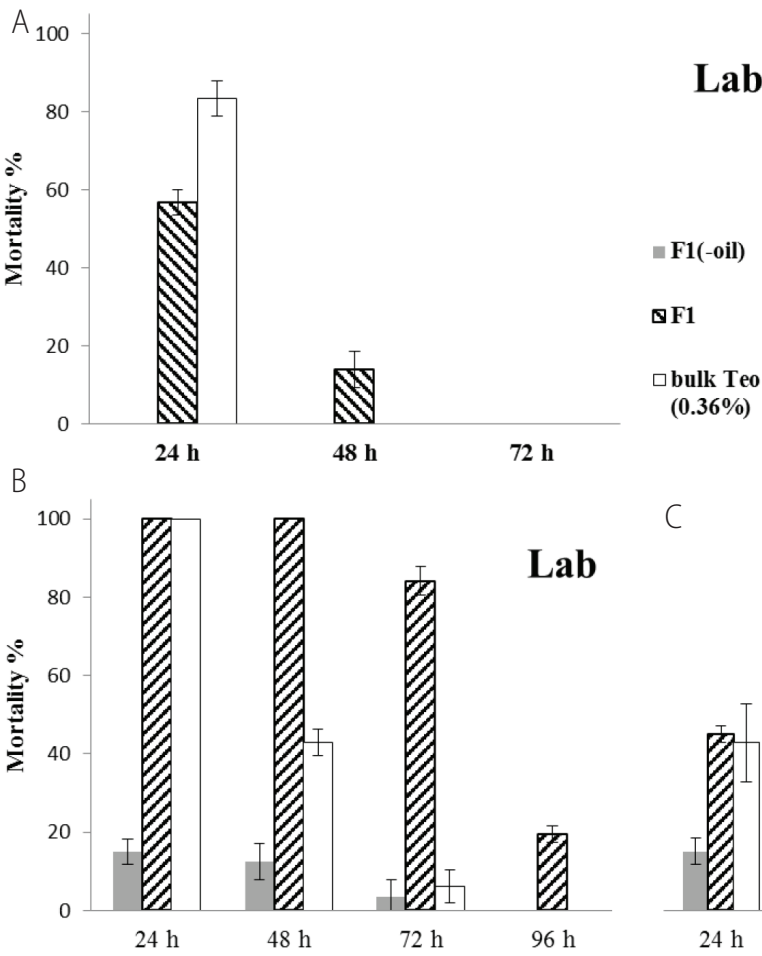

D

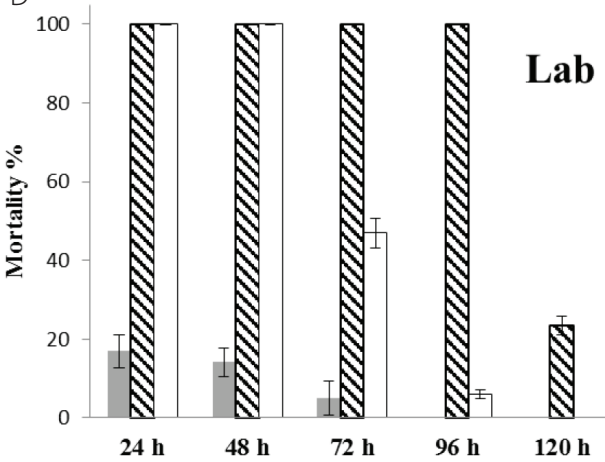

ab
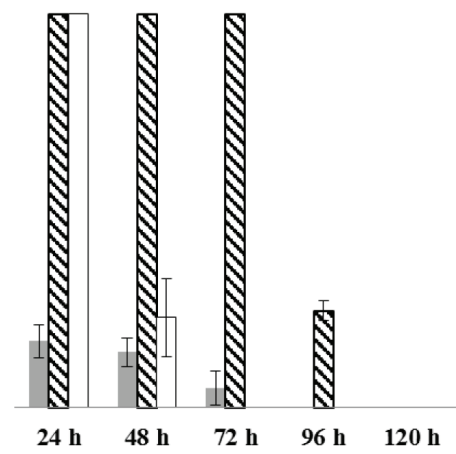

SSF

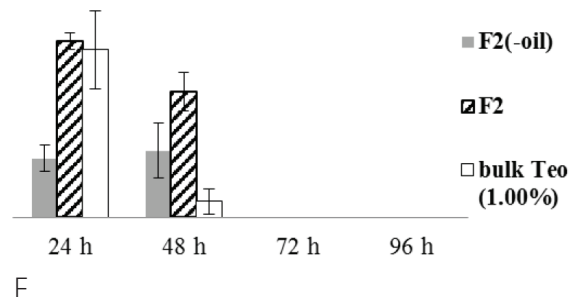

SSF

ॠ3(-oil)

QF3

$\square$ bulk Teo

$(1.60 \%)$
Fig. 5 Comparison of duration of larvicidal activities of selected nanoformulation vs. bulk TEO in the equal concentration of essential oil and also nanoformulations without oil both in lab and SSF tests. The final concentrations of TEO in the tests containers are: $(A) 18$ $\mu \mathrm{g} / \mathrm{mL}$, (B and C) $50 \mu \mathrm{g} / \mathrm{mL}$, (D and E) $80 \mu \mathrm{g} / \mathrm{mL}$. TEO: tarragon essential oil, SSF: simulated semi-field.

\section{Comparison of the Concentration of TEO during Larviciding Test}

The concentration of available TEO in the solutions during larvicidal bioassays for the samples, bulk TEO and optimum nanoformulation (F3) is shown in Fig. 6. In general, the rate of decrease in concentration of oil in bulk TEO in lab test $(y=-0.6012 x+60.905)$ is lower than that of SSF test $(y=-0.7333 x+55.4)$. This phenomenon is also observed in F3: related line equation in lab and SSF tests are $y=-0.4479 x$ +61.393 and $y=-0.5286 x+58.048$, respectively. These are due to harsh conditions such as higher temperature and air circulation which make higher evaporation of TEO in SSF. On the other hand, the decreasing rate in TEO concentration is slower in F3 compared with bulk TEO in both lab and SSF.

As the details show, bulk TEO shows a higher TEO concentration at the beginning, followed by a sharp drop in TEO concentration, in both lab and SSF tests. However, chitosan serves as an oil reservoir. Thus, it provides a sustained release of TEO, which in turn increases TEO concentration. This makes a longer duration of action for the nanoformulation compared with bulk TEO.
Interestingly, TEO concentrations are comparable with the activity of samples at a lab test (see Fig. 5D). For instance, the concentration of bulk TEO at $72 \mathrm{~h}$ in the lab was $11 \mu \mathrm{g} / \mathrm{mL}$, close to $\mathrm{LC}_{50}$ of TEO in lab test $(11.36 \mu \mathrm{g} / \mathrm{mL})$ and the LA observed was $47 \%$. However, in SSF test (Fig. 5E), the concentration and LA values do not agree. For instance, the concentration of bulk TEO at $24 \mathrm{~h}$ (SSF) was $19 \mu \mathrm{g} / \mathrm{mL}$ and its LA was $100 \%$. While, according to Fig. 1 , the lowest concentration to have a perfect LA should be $80 \mu \mathrm{g} / \mathrm{mL}$. In Fig. 5E, expected LA for this concentration (i.e. $19 \mu \mathrm{g} / \mathrm{mL}$ ) occurred at $48 \mathrm{~h}$ (i.e. $22 \%$ in SSF). It is, therefore, arguably that in SSF test, LA strongly depends on the concentration of TEO at the beginning time of exposure. In other words, the perfect LA which was observed at $24 \mathrm{~h}$ was due to the concentration of bulk TEO at zero time rather than $24 \mathrm{~h}$ after the start of the test.

Furthermore, according to Figs. 1, 5E, and 6, LA of F3 significantly improved compared with bulk TEO at SSF (independent-samples $t$-test, $P<0.05$ ): Lowest concentration of bulk TEO with full LA at SSF was $80 \mu \mathrm{g} / \mathrm{mL}$; while F3 at $48 \mathrm{~h}$ with $31 \mu \mathrm{g} / \mathrm{mL}$ concentration of TEO showed $100 \% \mathrm{LA}$ (see results of $72 \mathrm{~h}$ in Fig. 5E). It is, therefore, arguable that, by 


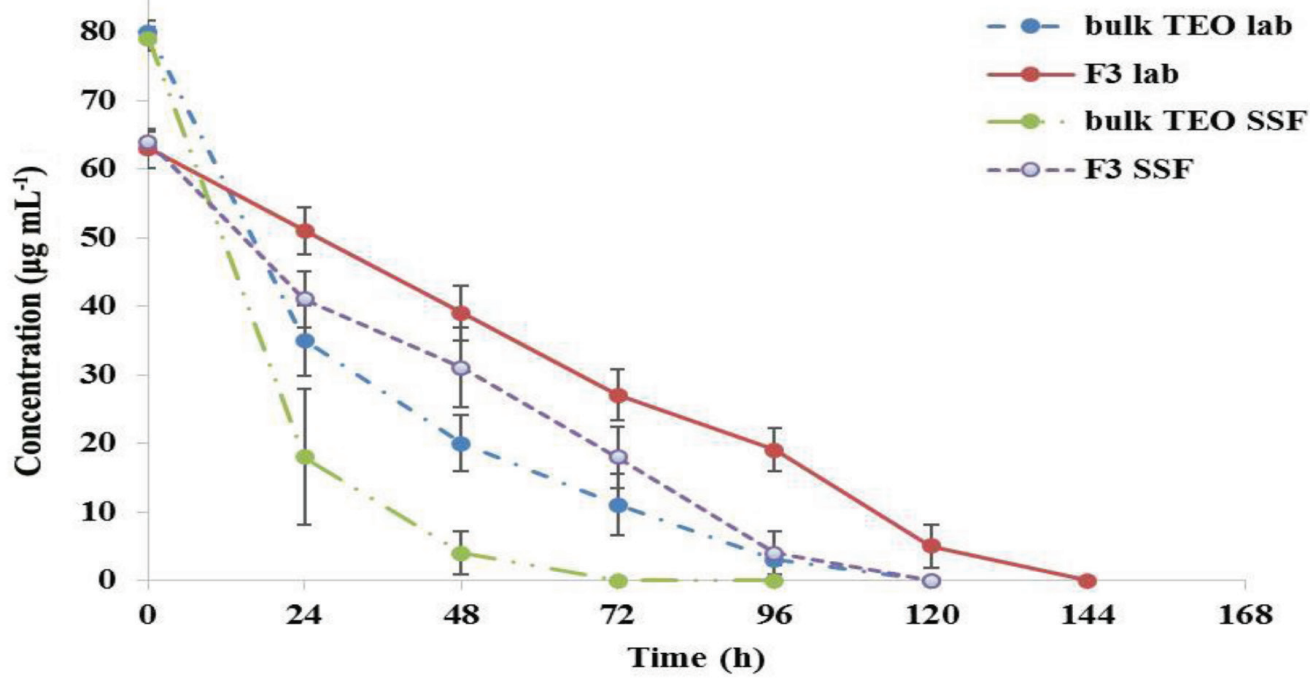

Lab

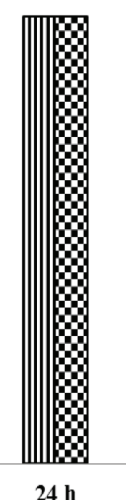

SSF
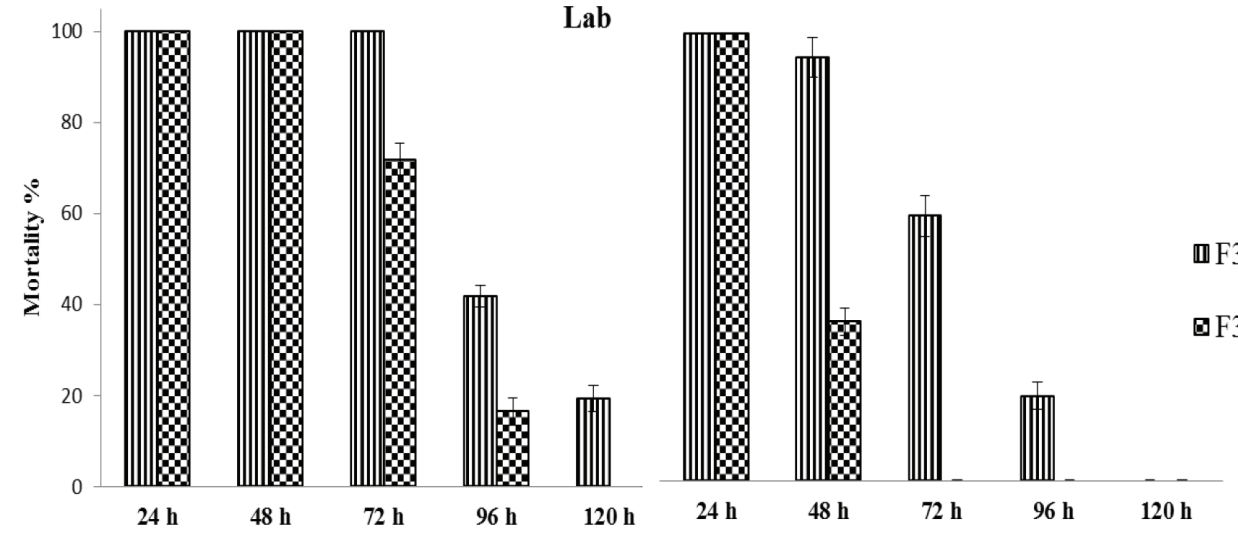

Fig. 6 Comparison of the concentration of bulk TE0 (1.6\% essential oil) and F3 (nanoformulation with $1.6 \%$ essential oil) during the larviciding test in the lab and simulated semi-field (SSF) tests. TE0: tarragon essential oil.

Fig. 7 Evaluation of the larvicidal activity of non-encapsulated F3 [F3(-TPP)] and microformulation [F3(micro)], both in the lab (left) and simulated semi-field (SSF) (right) test. The final concentration of TEO in the tests containers is $80 \mu \mathrm{g} / \mathrm{mL}$. TPP: tripolyphosphate, TEO: tarragon essential oil. using nanoformulation of TEO, not only durability of LA increases, but the efficacy rises too. No report has been found indicating that monitoring concentration of any EO during the larvicidal bioassays and evaluating related LA was performed simultaneously.

\section{Evaluating the Encapsulation Effect and the Size of the Capsules on the Duration of LA}

Figure 7 reports the LA of F3 in non-encapsulated form i.e. when no TPP was added to encapsulate the TEO: F3(-TPP) and when the particle size was in the range of micro [i.e. $850 \pm$ $35 \mathrm{~nm}, \mathrm{~F} 3$ (micro)] in both lab and SSF tests. The results show that perfect LA of F3(-TPP) and F3(micro) remain for 2 and 3 days in lab tests, respectively, which are significantly lower than that of F3 i.e. 4 days, (one-way ANOVA, $P<0.05$ ). These values decrease to 1 and 2 days in SSF test, respectively which are significantly smaller than that of F3 i.e. 3 days (one-way ANOVA, $P<0.05)$.

Interestingly, LA profile of F3(-TPP) is similar to that of bulk TEO (1.60\%) in Fig. 5D. Considering the fact that ingredient in F3(-TPP) was similar to F3 except for the presence of TPP which is necessary to prepare chitosan nanocapsules, the important role of encapsulation of TEO is clear. Whereby non-encapsulated TEO acts similar to bulk TEO. Since TPP had no LA (Data not given), the preparation of chitosan nanocapsules is the reason for the significant changes in LA of F3 and F3(-TPP) samples (independent-samples $t$-test, $P<0.05$ ).
Furthermore, to evaluate the effect of particle size, LA of F3 was compared with its corresponding microformulation preparation [i.e. F3(micro)], with same ingredients concentration, just with a larger particle size (i.e. $850 \pm 35 \mathrm{~nm}$ ). The duration of LA of F3(micro) was significantly lower than F3 (independent-samples $t$-test, $P<0.05$ ), most probably due to higher precipitation of microformulation compared to nanoformulation, as we noted during the tests. In other words, precipitation instead of evaporation removed TEO from the solutions, thus, less activity was observed. It was mentioned that larvae of $A$. stephensi normally breathe on the surface of the water and avoids the bottom of the container that contained precipitated microparticles..$^{35}$ This reduces the chance of physical interactions between larvae and the preparation containing TEO.

These results were in line with a report in which essential oil of $L$. sidoides was loaded in Alginate/cashew gum beads in two forms [i.e. floating and non-floating beads $(\sim 1.7 \mathrm{~mm})]$. Floating beads showed very good buoyancy up to 6 days and its LA after $48 \mathrm{~h}$ was around $85 \%$ against A. aegypti, while corresponded mortality to non-floating beads was around $30 \%{ }^{14}$

\section{Conclusion}

Encapsulated TEO into chitosan-TPP nanocapsules showed a longer duration of action and also higher efficacy in the lab 
and simulated semi-field tests compared with bulk TEO, non-encapsulated TEO, and chitosan microcapsules. The nanocapsules were able to protect the TEO from evaporation to provide long-lasting LA. As investigated, there is a logical relationship between LA and the concentration of TEO in different hours. The possibility of scale-up has been realized by using the very fast and facile method to nanoencapsulation of TEO. Based on the points mentioned, this formulation could be an interesting alternative to industrial larvicides.

\section{Acknowledgments}

This research was supported by Tehran University of Medical Sciences \& Health Services grant No. 95-01-87-31860, and also had ethical approval by its ethical committee centre, IR. TUMS.REC.1395.2480.

\section{Conflict of Interest}

There is no conflict of interest among the authors.

\section{References}

1. WHO. World Malaria Report 2016, 2016. Available from: http://www.who. int/malaria/publications/world-malaria-report-2016/report/en/.

2. Bellan SE. The importance of age dependent mortality and the extrinsic incubation period in models of mosquito-borne disease transmission and control. PLoS One. 2010;5:e10165.

3. Sumitha KV, Thoppil JE. Larvicidal efficacy and chemical constituents of O. gratissimum L. (Lamiaceae) essential oil against Aedes albopictus Skuse (Diptera: Culicidae). Parasitol Res. 2016;115:673-680.

4. Osanloo M, Sereshti H, Sedaghat MM, Amani A. Nanoemulsion of Dill essential oil as a green and potent larvicide against Anopheles stephensi. Environ Sci Pollut Res Int. 2018;25:6466-6473.

5. Peiris HTR, Hemingway J. Temephos resistance and the associated crossresistance spectrum in a strain of Culex quinquefasciatus Say (Diptera: Culicidae) from Peliyagoda, Sri Lanka. Bull Entomol Res. 1990;80:49-55.

6. Wirth MC, Georghiou GP. Selection and characterization of temephos resistance in a population of Aedes aegypti from Tortola, British Virgin Islands. J Am Mosq Control Assoc. 1999;15:315-320

7. Melo-Santos MA, Varjal-Melo JJ, Araújo AP, Gomes TC, Paiva MH, Regis LN, et al. Resistance to the organophosphate temephos: mechanisms, evolution and reversion in an Aedes aegypti laboratory strain from Brazil. Acta Trop. 2010;113:180-189.

8. Rodríguez MM, Bisset J, Ruiz M, Soca A. Cross-resistance to pyrethroid and organophosphorus insecticides induced by selection with temephos in Aedes aegypti (Diptera: Culicidae) from Cuba. J Med Entomol. 2002;39:882-888.

9. Soltani A, Vatandoost $H$, Oshaghi MA, Ravasan NM, Enayati AA, Asgarian F. Resistance mechanisms of Anopheles stephensi (Diptera: Culicidae) to Temephos. J Arthropod Borne Dis 2015:9:71-83.

10. Vatandoost $H$, Hanafi-Bojd AA. Current resistant status of Anopheles stephensi liston to different larvicides in Hormozgan province, southeastern Iran, 2004. Pak J Biol Sci. 2005;8:1568-1570.

11. Vatandoost H, Mashayekhi M, Abaie MR, Aflatoonian MR, Hanafi-Bojd AA, Sharifi I. Monitoring of insecticides resistance in main malaria vectors in a malarious area of Kahnooj district, Kerman province, southeastern Iran. J Vector Borne Dis. 2005:42:100-108.

12. Li J, Li Y, Dong H. Controlled release of herbicide acetochlor from clay/carboxylmethylcellulose gel formulations. J Agric Food Chem. 2008:56:1336-1342.

13. Maji TK, Baruah I, Dube S, Hussain MR. Microencapsulation of Zanthoxylum limonella oil (ZLO) in glutaraldehyde crosslinked gelatin for mosquito repellent application. Bioresour Technol. 2007:98:840-844.

14. Paula HC, de Oliveira EF, Abreu FO, de Paula RC. Alginate/cashew gum floating bead as a matrix for larvicide release. Mater Sci Eng C Mater Biol Appl. 2012:32:1421-1427.

15. Badawy MEI, Taktak NEM, Awad OM, Elfiki SA, El-Ela NEA. Larvicidal activity of temephos released from new chitosan/alginate/gelatin capsules against Culex pipiens. Int J Mosq Res. 2015;2:45-55.

16. Bhan S, Mohan L, Srivastava CN. Relative larvicidal potentiality of nanoencapsulated Temephos and Imidacloprid against Culex quinquefasciatus. J Asia Pac Entomol. 2014;17:787-791.

17. Sanei-Dehkordi A, Sedaghat MM, Vatandoost H, Abai MR. Chemical compositions of the peel essential oil of Citrus aurantium and its natural larvicidal activity against the malaria vector Anopheles stephensi (diptera: culicidae) in comparison with Citrus paradisi. J Arthropod Borne Dis. 2016;10:577-585.

18. Osanloo M, Amani A, Sereshti H, Shayeghi M, Sedaghat MM. Extraction and chemical composition essential oil of Kelussia odoratissima and comparison

its larvicidal activity with Z-ligustilide (Major Constituent) against Anopheles stephensi. J Entomol Zool Stud. 2017;5:611-616.

19. Vivekanandhan P, Senthil-Nathan S, Shivakumar MS. Larvicidal, pupicidal and adult smoke toxic effects of Acanthospermum hispidum (DC) leaf crude extracts against mosquito vectors. Physiol Mol Plant Pathol. 2018;101:156-162.

20. Jinu U, Rajakumaran S, Senthil-Nathan S, Geetha N, Venkatachalam P. Potential larvicidal activity of silver nanohybrids synthesized using leaf extracts of Cleistanthus collinus (Roxb.) Benth. ex Hook. f. and Strychnos nux-vomica L. nux-vomica against dengue, Chikungunya and Zika vectors. Physiol Mol Plant Pathol. 2018;101:163-171.

21. Yang FL, Li XG, Zhu F, Lei CL. Structural characterization of nanoparticles loaded with garlic essential oil and their insecticidal activity against Tribolium castaneum (Herbst) (Coleoptera: Tenebrionidae). J Agric Food Chem. 2009;57:10156-10162.

22. Duarte JL, Amado JRR, Oliveira AEMFM, Cruz RAS, Ferreira AM, Souto RNP, et al. Evaluation of larvicidal activity of a nanoemulsion of Rosmarinus officinalis essential oil. Rev Bras Farmacogn. 2015;25:189-192.

23. Sugumar S, Clarke SK, Nirmala MJ, Tyagi BK, Mukherjee A, Chandrasekaran N. Nanoemulsion of eucalyptus oil and its larvicidal activity against Culex quinquefasciatus. Bull Entomol Res. 2014;104:393-402.

24. Rodrigues ECR, Ferreira AM, Vilhena JCE, Almeida FB, Cruz RAS, Florentino $A C$, et al. Development of a larvicidal nanoemulsion with Copaiba (Copaifera duckei) oleoresin. Rev Bras Farmacogn. 2014;24:699-705.

25. Ghosh V, Mukherjee A, Chandrasekaran N. Formulation and characterization of plant essential oil based nanoemulsion: evaluation of its larvicidal activity against Aedes aegypti. Asian J Chem. 2013;25:S321-S323.

26. Anjali CH, Sharma Y, Mukherjee A, Chandrasekaran N. Neem oil (Azadirachta indica) nanoemulsion-a potent larvicidal agent against Culex quinquefasciatus. Pest Manag Sci. 2012;68:158-163.

27. Shahriyary L, Yazdanparast R. Inhibition of blood platelet adhesion, aggregation and secretion by Artemisia dracunculus leaves extracts. J Ethnopharmacol. 2007;114:194-198.

28. Sayyah M, Nadjafnia L, Kamalinejad M. Anticonvulsant activity and chemical composition of Artemisia dracunculus L. essential oil. J Ethnopharmacol. 2004;94:283-287

29. Osanloo M, Amani A, Sereshti H, Abai MR, Esmaeili F, Sedaghat MM Preparation and optimization nanoemulsion of Tarragon (Artemisia dracunculus) essential oil as effective herbal larvicides against Anopheles stephensi. Ind Crops Prod. 2017;109:214-219.

30. WHO. Guidelines for laboratory and field testing of mosquito larvicides 2005. Available from: http://apps.who.int/iris/handle/10665/69101.

31. Hosseini SF, Zandi M, Rezaei M, Farahmandghavi F. Two-step method for encapsulation of oregano essential oil in chitosan nanoparticles: preparation, characterization and in vitro release study. Carbohydr Polym. 2013;95:50-56.

32. Mohammadi A, Hashemi M, Hosseini SM. Nanoencapsulation of Zataria multiflora essential oil preparation and characterization with enhanced antifungal activity for controlling Botrytis cinerea, the causal agent of gray mould disease. Innov Food Sci Emerg Technol. 2015;28:73-80.

33. Esmaeili A, Asgari A. In vitro release and biological activities of Carum copticum essential oil (CEO) loaded chitosan nanoparticles. Int J Biol Macromol. 2015;81:283-290

34. Paula HCB, Sombra FM, de Freitas Cavalcante R, Abreu FOMS, de Paula RCM. Preparation and characterization of chitosan/cashew gum beads loaded with Lippia sidoides essential oil. Mater Sci Eng C. 2011:31:173-178.

35. Nation JL Sr. Insect Physiology and Biochemistry. CRC Press; 2008.

This work is licensed under a Creative Commons Attribution-NonCommercial 3.0 Unported License which allows users to read, copy, distribute and make derivative works for non-commercial purposes from the material, as long as the author of the original work is cited properly. 